\title{
Travaux sur la censure et les index des livres interdits réalisés à l'Université de Sherbrooke
}

\author{
J. M. DE BUJANDA
}

Université de Sherbrooke

\begin{abstract}
L'invention de l'imprimerie au XVe siècle savè̀re un puissant facteur de diffusion des idées dont se servent les autorités civiles et religieuses ainsi que le mouvement humaniste. Quand au XVI siècle limprimerie devient le principal moyen de diffusion de la Réforme protestante, les autorités ecclésiastiques et civiles qui restent fidèles à l'Église romaine essaient d'empêcher l'impression, la vente, la possession et la lecture des ouvrages hétérodoxes. C'est ainsi qu'on assiste à la parution des premières listes des ouvrages et des auteurs condamnés, désignés comme index des livres interdits. Cette pratique de la Contre-réforme officialisée par le Concile de Trente est d'une importance toute particulière au XVI e siècle. Elle devient par la suite une arme importante pour défendre le catholicisme contre ses ennemis extérieurs et intérieurs et se perpétue jusquau concile Vatican II, au milieu du XXe siècle. Pendant une quarantaine d’années, le Centre d'Études de la Renaissance de l'Université de Sherbrooke a consacré de nombreuses recherches à létude des index du XVI siècle et des index postérieurs publiés par l'Inquisition espagnole et par les Congrégations romaines de l'Index et du Saint-Office. Ces recherches ont donné lieu à plusieurs publications dont les 12 volumes de la collection Index des livres interdits.
\end{abstract}

The invention of printing in the fifteenth century was an important factor in the dissemination of ideas that served civic and religious authorities as well as the humanist movement. When, in the sixteenth century, printing became the primary means of spreading the Protestant Reformation, civic and ecclesiastical authorities who remained faithful to the Roman Catholic church attempted to halt the printing, sale, possession, and reading of heterodox works. It is thus that we witness the birth of the first lists of forbidden books and authors, which were given the title "Index of Prohibited Books." This Counter-Reformation practice, formalized by the Council of Trent, was of particular importance in the sixteenth century. It became, over time, an important means of defending Catholicism against its external and internal enemies, and the practice continued until Vatican II in the mid-twentieth century. Over the past four decades, the Centre d'Études de la Renaissance de l'Universite de Sherbrooke has devoted many studies to the indices of the sixteenth century and to those published later by the Spanish Inquisition and by the Roman Curia's Congregations for the Index and for the Holy Office. This research has produced many publications, in particular the twelve volumes of the collection Index des livres interdits.

es recherches et les publications sur la censure et les index des livres interdits
réalisées au Centre d'études de la Renaissance et au département d'histoire 
de l'université de Sherbrooke se sont prolongées pendant une quarantaine d'années. Elles débutent avec la publication « La censure littéraire en Espagne au XVI ${ }^{\text {e }}$ siècle » dans le Canadian Journal of History, VII (1972), p. 1-15, et sont complétées en 2014 avec le livre Indice de libros prohibidos y espurgados de la Inquisición española, actuellement sous presse. Dans les premières années, nous avons étudié la censure ecclésiastique à partir de la matière censurée, c'est-à-dire du contenu des différents index des livres interdits et des index expurgatoires, et ce, à partir de la naissance de l'index au milieu du XVI ${ }^{\mathrm{e}}$ siècle. À partir de l'année 1996, moment où nous avons personnellement eu la permission de travailler aux Archives du Saint-Office à Rome, et surtout depuis 1998, année de l'ouverture de ces Archives aux chercheurs, nos travaux ont porté en grande partie sur la documentation nouvellement disponible. Nous avons aussi intensifié nos recherches dans le Fonds Inquisition des Archives de Madrid.

Cette recherche sur les index des livres interdits a été la plus significative du Centre d'études de la Renaissance à partir de 1982 quand nous avons obtenu une importante subvention du Conseil de Recherche en Sciences Humaines du Canada dans le cadre du programme Subventions aux grands travaux d'édition. À cette subvention qui a été renouvelée à plusieurs reprises, se sont ajoutées celles du programme Killam du Conseil des Arts du Canada, du Fonds FCAR (Formation des chercheurs et l'aide à la recherche) du Ministère de l'enseignement supérieur du Québec et celles de l'Université de Sherbrooke dans le cadre de son programme Subventions aux groupes d'excellence.

Les travaux ont été orientés et dirigés par un comité d'édition formé des professeurs J. M. De Bujanda, Peter G. Bietenholz, Serge Lusignan, Claude Sutto et John Tedeschi. Les membres de l'équipe qui ont travaillé au projet sont René Davignon, Ela Stanek, Marcella Richter et Claire Brochu ainsi que de nombreux étudiants, entre autres Pierre Cameron, Lyne Gagné, René Paquin, Jeanne Mance Rodrigue et Lucie Tanguay.

Même si la censure de l'Église remonte aux temps apostoliques et s'exerce pendant tout le Moyen-Âge, c'est après l'implantation de l'imprimerie au cours de la deuxième moitié du $\mathrm{XV}^{\mathrm{e}}$ siècle qu'on assiste à une organisation systématique de la censure. En recueillant des dispositions antérieures d'Innocent VIII et d'Alexandre VI destinées aux provinces ecclésiastiques de la région de Mayence, berceau de l'imprimerie, le $\mathrm{V}^{\mathrm{e}}$ Concile du Latran par la constitution Inter sollicitudines promulgue en 1515 une loi universelle qui 
établit l'exercice de la censure préalable à l'impression et le contrôle des écrits déjà publiés.

Quelques années plus tard, après la révolte de Martin Luther en 1517, l'imprimerie devient le principal moyen de diffusion de la Réforme. L'avalanche des pamphlets et des livres réformés provoque alors la réaction de ceux qui restent fidèles à l'Église romaine et qui essaient d'empêcher par tous les moyens l'impression, la vente, la possession et la lecture des ouvrages des réformateurs. On assiste ainsi à la parution de plusieurs listes d'ouvrages et d'auteurs condamnés qui émanent des universités, des autorités ecclésiastiques et civiles, et des inquisitions locales.

Le tout premier index des livres interdits imprimés est celui de la Faculté de Théologie de l'Université de Paris paru en 1544. Il est suivi par ceux de l'Université de Louvain et des inquisitions de Venise, du Portugal et d'Espagne. Rome publie son premier catalogue en 1559. Cinq ans plus tard, en 1564, le Concile de Trente adopte une version plus modérée en ajoutant dix règles générales qui encadrent l'exercice de la censure.

À partir du Concile de Trente, l'index des livres interdits occupe une place de première importance parmi les institutions de la Contre-Réforme. D'une part, on combat les points fondamentaux des théories des réformés par des écrits polémiques et on organise des campagnes de propagande et d'endoctrinement qui visent à renforcer la foi traditionnelle. En même temps, on veut empêcher par tous les moyens l'impression et la diffusion des écrits qui professent la nouvelle doctrine. Cette double attitude que canalise le Concile de Trente se concrétise en partie par la publication du catéchisme et de l'index des livres interdits. Le catéchisme est l'instrument d'endoctrinement et l'œuvre de référence par excellence de la foi et de la morale catholique. L'index des livres interdits, dont le but premier était de combattre l'hérésie protestante, est par la suite institutionnalisé par Rome qui en fait une arme importante pour se défendre de ses ennemis extérieurs et intérieurs qui menacent l'homogénéité de la foi catholique et le maintien des institutions ecclésiastiques.

La censure ne s'exerce pas uniquement sur les affaires religieuses. La foi est une notion fluide que le magistère ecclésiastique précise dans le temps, à mesure qu'il définit les dogmes et se prononce sur le contenu du message évangélique et ses conséquences. Considérée comme dépositaire de la Révélation et interprète authentique et autorisée, non seulement de la loi divine, mais aussi de la loi naturelle, l'Église peut se prononcer sur toute l'activité humaine. L'exercice de 
la censure peut varier par conséquent selon l'interprétation qu'on donne à la foi catholique et à la moralité humaine. Instrument de contrôle religieux, culturel et idéologique, l'index des livres interdits modifie son champ d'action en s'adaptant aux différentes conjonctures historiques et en étendant progressivement son activité censoriale à toutes les manifestations de la vie intellectuelle et sociale. Le concept d'orthodoxie devient plus rigide et plus englobant, débordant du terrain religieux et envahissant la politique, la philosophie, l'art, les manifestations de la vie quotidienne et le domaine proprement scientifique.

L'index étant une loi ecclésiastique, son application dépendait du pouvoir de coercition dont disposaient les autorités ecclésiastiques et de la collaboration que leur offraient les autorités civiles. En principe elle était observée dans les territoires où l'Inquisition était implantée solidement et dans les États pontificaux.

La censure et l'index ont certainement ralenti la production littéraire. Une directive de l'Inquisition imposait à tous l'obligation de dénoncer les livres et les propositions qui, à leur avis, pouvaient être contraires à la foi et aux bonnes mœurs. Dans ce climat de dénonciation et de soupçon, l'autocensure ne pouvait qu'empêcher l'expression d'idées originales. En apprenant la condamnation du livre de Galilée, René Descartes retire son manuscrit du Traité du monde en écrivant: " Le désir que j'ai de vivre en repos m'impose de garder pour moi mes théories ${ }^{1} »$. Plusieurs catholiques libéraux du XIX ${ }^{\mathrm{e}}$ siècle, comme Lord Acton et Franz Xavier Kraus, s'autocensurent et renoncent à écrire des ouvrages projetés.

Les comptes rendus critiques parus à la suite de la publication des différents volumes de notre collection dans de nombreuses revues ${ }^{2}$ ont bien mis en relief leur importance et leur utilité pour les spécialistes de différentes disciplines comme les historiens, les théologiens, les littéraires, les philosophes, les spécialistes des théories politiques, des courants scientifiques et beaucoup d'autres.

L'étude du contenu des index offre des pistes de recherche de première importance à tous ceux qui s'intéressent à l'histoire des mentalités, à l'évolution des valeurs, au changement et à la persistance des mœurs et des

1. Georges Minois, Censure et Culture sous l'Ancien Régime (Paris : Fayard, 1995), 129.

2. Voir par exemple : Paul F. Grendler, Canadian Journal of History / Annales canadiennes d'histoire 20.3 (1985) : 420-421, Michel Reulos, Revue historique du droit français et étranger 65 (1987) : 649-650, JeanPierre Dedieu, Bulletin Hispanique 88.1-2 (1986) : 244-248, Agostino Borromeo, The Catholic Historical Review 74.4 (1988) : 610-612, Rita Belladonna, Quaderni d'italianistica 11.1 (1990), p. 147-9. 
comportements. Les ouvrages interdits sont souvent des pointes d'iceberg qui nous conduisent vers des pistes d'exploration nouvelles et nous montrent les courants souterrains d'une anti-culture réprimée.

Voici un bref rappel du contenu des 12 volumes de la collection Index des livres interdits. D’autres publications ont aussi été réalisées à Sherbrooke.

\section{Collection Index des livres interdits}

I. Index de l'Université de Paris, 1544, 1545, 1547, 1548, 1551, 1556, par J. M. De Bujanda, James K. Farge et Francis Higman (Sherbrooke, Genève : Centre d'études de la Renaissance, Droz, 1985), 672 p.

Le tout premier index des livres interdits imprimés est celui de la Faculté de théologie de l'Université de Paris paru en 1544. À partir des condamnations prononcées depuis le début des années 1520, et surtout avec l'ajout de nouvelles interdictions, les théologiens de la Sorbonne élaborent une liste de 230 titres de livres en latin et en français qui sont prohibés. Le nombre des condamnations augmente graduellement dans les éditions subséquentes du catalogue publiées en 1545, 1547, 1549, 1551 et 1556. Dans la dernière édition du catalogue, on compte 528 interdictions dont 278 ouvrages en latin et 250 en français. Les écrits prohibés sont pour la plupart des traités théologiques, polémiques, didactiques ou catéchistiques de réformateurs parmi lesquels figurent les ouvrages littéraires d'Érasme et de Rabelais. Si la très grande majorité des écrits en latin sont condamnés avec nom d'auteur, près des deux tiers des écrits en français qui sont interdits sont anonymes.

II. Index de l'Université de Louvain, 1546, 1550, 1558, par J. M. De Bujanda et Léon-E. Halkin, avec la collaboration de Patrick Pasture et Geneviève Glorieux (Sherbrooke, Genève : Centre d'études de la Renaissance, Droz, 1986), 588 p.

Les théologiens de l'Université de Louvain, en se conformant aux ordres de Charles Quint et de Philippe II, publient trois index de livres interdits au cours des années 1546,1550 et 1558. Les 450 condamnations des catalogues de Louvain se rapportent à des écrits qui favorisent en général la diffusion de la Réforme. Ils sont regroupées en cinq sections : 60 éditions de la Bible et du Nouveau Testament, un peu moins de 300 écrits en latin, près de 90 en 
flamand parmi lesquels 5 sont en allemand et 14 en français. Les écrits en flamand présentent un intérêt particulier car plusieurs d'entre eux sont de petits opuscules de 8 à 10 folios in- $8^{\circ}$ destinés à diffuser les idées de la Réforme parmi le peuple; ils se présentent le plus souvent sans nom d'auteur, ni d'imprimeur, ou encore avec une fausse adresse typographique. Une autre particularité des catalogues de Louvain est l'existence d'une liste des livres permis dans les écoles qui impose un contrôle sur l'enseignement.

III. Index de Venise, 1549, et de Venise et Milan, 1554 par J. M. De Bujanda et Paul F. Grendler (Sherbrooke, Genève : Centre d'études de la Renaissance, Droz, 1987), $528 \mathrm{p}$.

Le premier catalogue des livres interdits imprimé en Italie paraîtà Venise en 1549. Il a été préparé d'un commun accord par l'Inquisition, le nonce apostolique et les «Tre Savii sopra Eresia » comme représentants de la «Signoria » de Venise. Moins précis que les index des Universités de Paris et de Louvain, ce catalogue comprend 149 interdictions dont un tiers qui se réfèrent à des auteurs dont on condamne tous les écrits, un tiers à des ouvrages particuliers avec nom d'auteur et l'autre tiers à des écrits anonymes. La forte opposition suscitée par ce catalogue chez les libraires et les imprimeurs conduit à sa suppression avant sa publication officielle.

Cinq ans plus tard, en 1554, on imprime à Milan, à Venise et à Florence un nouveau catalogue. L'index de Venise, un peu plus étendu que ceux de Milan et de Florence, contient tout près de 600 interdictions dont plus de la moitié, soit 332, qui visent la production entière d'auteurs, une centaine qui se réfèrent à des écrits particuliers avec nom d'auteur, et quelque 165 qui sont des titres anonymes avec quelques condamnations d'ordre général. De nouveau, les gens du livre et les intellectuels lancent une véritable offensive contre le catalogue, lequel, après six mois de durs affrontements entre ses promoteurs et leurs adversaires, est suspendu. Le catalogue imprimé à Florence en 1554, qui était inconnu jusqu'en 1996, date à laquelle nous l'avons trouvé aux Archives du Saint-Office, concorde fondamentalement avec les index de Venise et Milan.

IV. Index de l'Inquisition portugaise, 1547, 1551, 1561, 1564, 1581 par J. M. De Bujanda (Sherbrooke, Genève : Centre d'études de la Renaissance, Droz), 1995, $875 \mathrm{p}$. 
Les premiers index publiés par l'Inquisition portugaise s'inspirent et adoptent les catalogues des Universités de Paris et de Louvain. Le premier index portugais, promulgué en 1547 mais non imprimé, est avant tout un conglomérat de différentes listes d'écrits déjà condamnés. Plus de la moitié des 160 interdictions qu'on y trouve proviennent de la liste latine que les théologiens de Paris avaient publiée en 1544. Dans l'index portugais imprimé en 1551, la plus grande partie des quelque 500 condamnations qu'il renferme proviennent du catalogue publié par l'Université de Louvain l'année précédente.

À partir de la parution du premier index romain de 1559, l'Inquisition portugaise s'aligne essentiellement sur l'Inquisition romaine en faisant siennes ses condamnations ; elle va même plus loin que les directives romaines en ajoutant de nouvelles interdictions. Le premier index romain est réimprimé à Coimbra en 1559. Deux ans plus tard, en 1561, paraît le Rol dos livros defesos qui reproduit les condamnations de l'index de Paul IV et assume la grande majorité des interdictions des index antérieurs de l'Inquisition portugaise. L'index du Concile de Trente est réimprimé dès 1564 à Lisbonne, suivi d'un nouveau Rol des livres interdits au Portugal. Avec l'index de 1581, l'Inquisition portugaise reste fidèle à Rome en réimprimant l'index de Trente qu'elle fait suivre du Catálogo des livres interdits au Portugal. Ce dernier comprend une section en latin de 93 interdictions et une section en langue vulgaire de 107 condamnations, principalement en portugais et en castillan.

V. Index de l'Inquisition espagnole, 1551, 1554, 1559 par J. M. De Bujanda (Sherbrooke, Genève : Centre d'études de la Renaissance, Droz, 1984), 800 p.

Les quatre éditions connues du premier index espagnol parues en 1551 à Valladolid, Tolède, Séville et Valence, sont des rééditions de l'index de Louvain 1550 ; elles sont suivies d'un Memorial qui totalise 109 condamnations d'auteurs et de livres prohibés précédemment par le Saint-Office espagnol.

L'application des dispositions du catalogue de 1551 posait plusieurs problèmes, surtout concernant les éditions des Bibles interdites. L'Inquisition centrale nomme donc une commission pour l'expurgation d'un grand nombre d'éditions de la Bible et le résultat de ces travaux donne lieu à la publication de la Censura general de Biblias en 1554 qui ordonne l'expurgation de 65 éditions de l'Écriture sainte. 
En août 1559, huit mois après la publication du premier index romain, l'archevêque de Séville et Inquisiteur général Fernando de Valdés fait imprimer un important catalogue de livres interdits qui affirme l'indépendance de l'Inquisition espagnole à l'égard de l'Inquisition romaine. Les deux catalogues diffèrent considérablement dans le nombre et le degré des condamnations. Les inquisiteurs espagnols regroupent les 698 interdictions selon la langue des publications. Les sections de livres en latin, en flamand, en allemand, en français et en portugais dépendent fondamentalement des index de Louvain, de Paris et du Portugal, tandis que la section en castillan reflète les travaux de l'Inquisition espagnole qui se concentre principalement sur les livres de piété et de dévotion, les thèses érasmiennes et les pratiques superstitieuses.

VI.Index de l'Inquisition espagnole, 1583, 1584 par J. M. De Bujanda (Sherbrooke, Genève : Centre d'études de la Renaissance, Droz, 1993), 1246 p.

Dans les territoires de la Couronne espagnole, l'Inquisition possède une politique indépendante de Rome en matière de contrôle de la presse et publie ses propres catalogues. Celui de 1583 paraît après de longues années de préparation. Avec 2315 entrées regroupées selon la langue, le catalogue publié par le cardinal Gaspar de Quiroga est le plus volumineux de tous les index de livres interdits du XVI ${ }^{\text {e }}$ siècle. On y compte 1709 interdictions en latin, 197 en castillan, 18 en portugais, 72 en italien, 104 en français et 215 en flamand ou en allemand. L'index espagnol intègre la majorité des condamnations des index publiés par les autorités espagnoles à Anvers en 1570 et 1571 et par l'Inquisition portugaise en 1581. On regroupe les normes générales dans quatorze règles qui proviennent des règles de l'index romain et des dispositions adoptées par l'inquisition espagnole.

Comme complément au catalogue des livres interdits, on publie l'année suivante, en 1584, l'index des livres expurgés. En procédant ainsi, l'Inquisition espagnole répondait aux demandes des intellectuels, des professeurs d'université, des professionnels et aux plaintes des imprimeurs et des libraires. L'étendue des expurgations des 81 entrées principales s'étend sur 395 pages. Si on regarde le contenu des chapitres, paragraphes, phrases et mots supprimés, on constate que les corrections ont été introduites dans le but d'éliminer les points de vue contraires aux enseignements et aux prescriptions de l'Église 
catholique, les critiques faites aux institutions religieuses, et tout ce qui, d'une façon ou d'une autre, semble favorable à la Réforme protestante.

VII. Index d'Anvers, 1569-1571 par J. M. De Bujanda. Introduction historique de Léon-E. Halkin (Sherbrooke, Genève : Centre d'études de la Renaissance, Droz, 1987), $976 \mathrm{p}$.

En 1569 et 1570, dans les Pays-Bas espagnols, Fernando Alvarez de Toledo, duc d'Alba, ordonne l'impression de l'index de Trente avec un important appendice. Une commission présidée par Benito Arias Montano a rédigé l'appendice publié à Anvers en 1570 lequel compte plus de 800 condamnations avec des sections d'ouvrages en latin, en flamand, en français et en castillan. L'application des normes du catalogue et des dispositions promulguées par le décret royal de publication soulevait de nombreuses questions, surtout concernant la possibilité de conserver les ouvrages une fois expurgés.

Suite à une large consultation du milieu intellectuel, une nouvelle commission propose alors un catalogue des livres à expurger qui est publié à Anvers en 1571. Le travail d'expurgation visait à mettre en pratique une des dispositions des Pères du Concile de Trente permettant l'utilisation des écrits des auteurs de première classe dans la mesure où ceux-ci pouvaient être expurgés. Dans l'index expurgatoire d'Anvers, figurent 83 auteurs avec 207 ouvrages regroupés en six sections : théologie, droit, médecine, philosophie, mathématiques et humanités. Le résultat de cette chirurgie intellectuelle apparaît comme un moyen de récupération d'un secteur important de la culture européenne.

VIII. Index de Rome, 1559, 1564. Les premiers index romains et l'Index $d u$ Concile de Trente par J M. De Bujanda (Sherbrooke, Genève : Centre d'études de la Renaissance, Droz, 1990), 1039 p.

Après son établissement en 1542, l'Inquisition romaine prit en charge le contrôle de l'imprimerie, de la vente et de la diffusion des écrits. Les travaux de préparation d'un index romain s'intensifient à partir de 1555 quand l'Inquisiteur général Gian Pietro Caraffa devient pape avec le nom de Paul IV.

L'index paru en 1559, qui est une déclaration de guerre contre la littérature réformée, contient plus d'un millier d'interdictions divisées en 
trois classes. La première classe, totalisant 603 condamnations, comprend les auteurs qui se sont écartés de la foi catholique et dont on interdit tous les écrits même s'ils ne contiennent rien contre ou sur la religion. La deuxième classe regroupe les écrits particuliers avec nom d'auteur ; elle compte 126 titres prohibés qui appartiennent à 117 auteurs. Dans la troisième classe réservée aux écrits considérés comme anonymes, on condamne 332 titres. Ce catalogue romain contient aussi 45 interdictions de Bibles et de Nouveaux Testaments et une liste de 61 noms d'imprimeurs responsables de la publication de livres hérétiques. Mais plus encore que dans le nombre des condamnations, c'est dans certaines dispositions générales que se trouve la rigueur de l'index de Paul IV. Devant les pressions exercées et les demandes provenant tant des enseignants, des intellectuels, des autorités religieuses et politiques que des libraires, les inquisiteurs romains en viennent à la conclusion qu'il faut atténuer la rigueur de certaines règles. L'Instructio circa indicem de février 1559 et la Moderatio indicis de juin 1561 vont dans le sens de la modération.

L'index de Paul IV fut révisé par une commission du concile de Trente et promulgué ensuite par Pie IV en 1564. La vraie différence entre les catalogues de Paul IV et de Pie IV ne se trouve pas dans les condamnations particulières qui sont presque les mêmes dans les deux index, mais dans les dix règles qui formeront la loi cadre de la censure catholique pendant quatre siècles. Elles acceptent de plus l'exercice de l'expurgation comme une modalité de censure.

IX. Index de Rome, 1596. Avec étude des index de Parme, 1580, Munich, 1582 et de Rome 1590, 1593 par J. M. De Bujanda, Peter Bietenholz, Ugo Rozzo, et Paul Grendler ; textes traduits par Claude Sutto (Sherbrooke, Genève : Centre d'études de la Renaissance, Droz, 1994), 1174 p.

L'index de Munich de 1582 est une réimpression de l'index de Trente avec l'ajout de 346 noms d'auteurs condamnés, qui proviennent de Messkataloge des foires de Francfort. La presque totalité de ces condamnations sont ensuite assumées par l'index romain. Au cours des décennies 1570 et 1580, plusieurs inquisitions locales d'Italie comme celles de Parme, d'Alessandria et de Naples, ajoutent des suppléments à l'index. L'index imprimé à Parme en 1580 inclut de nombreux ouvrages en langue vulgaire qui abordent des sujets scabreux du point de vue de la morale post-tridentine, et est particulièrement révélateur d'un élargissement de la censure. 
Les index produits et imprimés à Rome en 1590 et 1593 ajoutent à l'index de Trente de très nombreuses condamnations d'auteurs et d'ouvrages en latin et en langue vulgaire, de même que plusieurs dispositions à caractère général qui élargissent et durcissent la pratique de la censure. Les réactions négatives qui s'ensuivent de la part de nombreux ecclésiastiques, intellectuels et libraires ainsi que l'opposition ferme d'autorités politiques, principalement de la Seigneurie de Venise, expliquent le retrait de ces deux index. Mais même si un nombre important de leurs condamnations, 265 en latin et 181 en italien, ne devinrent jamais effectives, elles nous révèlent les préoccupations et les projets d'un secteur important de la Curie romaine.

L'index publié finalement en 1596 par Clément VIII est une solution de compromis entre les différentes tendances qui s'affrontèrent tout au long du processus de son élaboration. Le nouvel index reproduit fidèlement le catalogue de Trente publié 32 ans auparavant, et ajoute en appendice 1143 condamnations : 682 auteurs dont toute la production est interdite, 185 écrits avec nom d'auteur et 276 ouvrages anonymes. Une particularité importante de l'index clémentin par rapport aux autres index romains, est quapproximativement la moitié des ouvrages avec nom d'auteur sont interdits en attendant d'être expurgés. Aux dix règles de l'index de Trente, Clément VIII ajoute des directives qui doivent être suivies dans la prohibition, la correction et l'impression des livres. L'Instructio de Clément VIII, qui servira de cadre de référence pour l'exercice de la censure jusqu'à la réforme de Léon XIII au début du $\mathrm{XX}^{\mathrm{e}}$ siècle, offre une réglementation précise sur la pratique de la censure préalable à l'impression et sur l'exercice des professions d'imprimeur, de libraire et de travailleur de l'imprimerie.

X. Thesaurus de la littérature interdite au XVI siècle par J. M. De Bujanda (Sherbrooke, Genève : Centre d'études de la Renaissance, Droz, 1996), 840 p.

Le volume offre une vision synthétique de la matière analysée dans les neuf premiers volumes. La première section présente les auteurs et ouvrages condamnés ou expurgés au $\mathrm{XVI}^{\mathrm{e}}$ siècle. Le nombre des auteurs dont les écrits sont condamnés en tout ou en partie s'élève à quelque 1900. De ces écrivains, il y en a à peu près 1400, appelés de première classe, dont tous les écrits sont interdits ; parmi ceux-ci, certains ont aussi des écrits prohibés nominalement. C'est le cas par exemple de Martin Luther qui, en plus d'être auteur interdit, compte 75 écrits pour la majorité anonymes, ou de l'humaniste Érasme de 
Rotterdam, auteur de première classe dans certains index dont on interdit explicitement 59 ouvrages.

Les ouvrages interdits de façon absolue ou en attendant d'être expurgés qui figurent dans le Thesaurus sans le nom des auteurs sont au nombre de 2150 . Parmi ceux-ci 460 sont à expurger. Les écrits prohibés dans les index comme anonymes sont environ 1100. Aujourd'hui on connaît la paternité d'à peu près la moitié de ces titres. Les autres sont des livres, des brochures, des prières ou d'autres sortes d'opuscules qui restent encore anonymes.

La deuxième partie du Thesaurus qui recense les éditions des ouvrages interdits nominalement, offre un aperçu intéressant des lieux et des imprimeurs qui ont principalement contribué à la diffusion des idées réformées. Les centaines ou les milliers d'éditions d'ouvrages interdits publiés dans des centres comme Bâle, Genève, Anvers ou Lyon contrastent fortement avec la trentaine d'éditions produites au Portugal. La chronologie de la production des éditions condamnées est aussi hautement significative et nous pouvons en tirer des conclusions sur l'ampleur de la diffusion du livre interdit.

XI. Index librorum peohibirorum, 1600-1696, par J. M. De Bujanda avec l'assistance de Marcella Richter (Sherbrooke, Genève : Centre d'études de la Renaissance, Médiaspaul, Droz, 1996), 980 p.

Ce catalogue contient tous les livres mis à l'index romain à partir de l'an 1600 jusqu'à son retrait en 1966. Aux condamnations qui se trouvent dans sa dernière édition de 1948, ont été ajoutés les autres livres interdits après cette date et les centaines de titres qui ont figuré dans l'index à un moment donné mais qui ont été retirés, principalement lors de la réforme de Léon XIII en 1900. Contrairement aux différentes éditions officielles de l'Index publiées au $\mathrm{XX}^{\mathrm{e}}$ siècle qui présentent uniquement le nom de l'auteur et le titre de l'ouvrage en abrégé sans aucune description bibliographique, ce volume offre dans la mesure du possible de courtes informations biographiques sur l'auteur et une brève description de l'édition princeps ou de la première édition connue.

À partir du début du XVII ${ }^{\mathrm{e}}$ siècle, la Congrégation de l'Index procède à l'interdiction de livres par décrets particuliers qui regroupent souvent ses condamnations et celles prononcées par le Saint-Office de l'Inquisition. À des intervalles différents paraissent des éditions de l'Index qui incorporent les contenus des décrets de condamnation. 
Le nombre total d'interdictions mis à l'index romain au cours de la période 1600-1966 est approximativement de 5200 ; le nombre peut varier légèrement selon les méthodes de comptage car une œuvre paraît parfois dans plusieurs langues et sous des titres différents. Les auteurs de ces ouvrages sont près de 3000 dont un peu plus de 200 sont des auteurs secondaires (adaptateurs, traducteurs, etc.). Pris d'une façon absolue, ces chiffres ne sont pas en soi très élevés. Il faut cependant tenir compte qu'un bon nombre d'autres titres tombaient sous les interdictions de règles générales de l'index et que les diocèses et les provinces ecclésiastiques exerçaient par eux-mêmes la censure et possédaient leurs propres catalogues.

XII. El Índice de libros prohibidos y expurgados de la Inquisición Española (15511819), par J. M. De Bujanda avec l'assistance de Marcella Richter (Sherbrooke, Madrid : Centre d'études de la Renaissance, BAC, 2014 [sous presse]), environ $1300 \mathrm{p}$.

En Espagne, la censure préventive antérieure à l'impression relevait de l'autorité royale tandis que la censure répressive postérieure à l'impression était exercée par l'Inquisition. Pendant les trois siècles et demi de son existence (1478-1834), le Saint-Office de l'Inquisition espagnole qui exerce l'activité de la censure des livres par de multiples procédés, publie neuf index de livres interdits et expurgés dont certains sont des volumes in folio qui dépassent le millier de pages. À partir de la publication du dernier index en 1790, les prohibitions figurent dans les édits postérieurs jusqu'en 1819. À partir de cette date, l'Index romain est en vigueur dans le territoire espagnol de la péninsule et dans ses colonies américaines, et les évêques exercent les pouvoirs que leur reconnaît le droit canonique en matière de censure.

La première partie du volume offre une vue d'ensemble sur l'exercice et l'évolution de la censure inquisitoriale en exposant son contenu et les modalités de l'élaboration des catalogues et des édits de prohibition et d'expurgation. On présente aussi une étude sur l'impact et les conséquences de la censure inquisitoriale sur la création littéraire et l'évolution de la religiosité, de la culture, des institutions et de la vie du peuple espagnol.

La seconde partie présente d'une manière concise la totalité des auteurs condamnés et des œuvres interdites ou expurgées, en signalant leur présence dans les index publiés entre 1551 et 1790 . Un appendice recueille les condamnations 
présentes dans les édits postérieurs. Le nombre d'auteurs et d'ouvrages interdits ou expurgés par les index et les édits de l'Inquisition espagnole approche 9000. 